\title{
Romiplostim for the Treatment of Immune Thrombocytopenia: Spotlight on Patient Acceptability and Ease of Use
}

This article was published in the following Dove Press journal: Patient Preference and Adherence

\section{Megan M Gilbert}

Amanda B Grimes (DD

Taylor Olmsted Kim

Jenny M Despotovic

Department of Pediatrics, Section of Hematology/Oncology, Baylor College of Medicine, Houston, TX, USA
Correspondence: Megan M Gilbert Department of Pediatrics, Section of Hematology/Oncology, Baylor College of Medicine, Texas Children's Hospital, 670 Fannin Suite 1510, Houston, TX 77030 USA

Tel +I (832) 824-4736

Fax +l (832) 825-4846

Email megan.gilbert@bcm.edu

\begin{abstract}
Immune thrombocytopenia (ITP) is an immune-mediated disorder resulting in platelet destruction and subsequent thrombocytopenia. Bleeding symptoms range from mild cutaneous bleeding to life-threatening hemorrhage. Romiplostim, a peptide-antibody fusion product, is a thrombopoietin receptor agonist (TPO-RA) indicated for use in patients with ITP. Romiplostim is US Food and Drug Administration (FDA) approved in children $\geq 1$ year of age with ITP of $>6$ months' duration who have had an inadequate response to first-line therapies or splenectomy. FDA approval in adults with chronic ITP was expanded in October 2019 to include adults with newly diagnosed ( $<3$ months' duration) and persistent (3-12 months' duration) ITP who demonstrated an inadequate response to first-line therapies, including corticosteroids and immunoglobulins, or splenectomy. The newly published 2019 American Society of Hematology ITP Guidelines place TPO-RAs, including romiplostim, as second-line therapies in both children and adults. Here, we review the use of romiplostim as second-line therapy with a spotlight on health-related quality of life, ease of use, and patient preference.
\end{abstract}

Keywords: romiplostim, immune thrombocytopenia, quality of life, patient preference

\section{Introduction}

Immune thrombocytopenia (ITP) is an acquired disorder resulting in immunemediated platelet destruction and isolated thrombocytopenia which affects 2 to 5 in 100,000 persons per year. ${ }^{1}$ The pathophysiology of ITP is highly complex and has not been fully elucidated to date. Accepted mechanisms include immune dysregulation caused by an often unidentified trigger leading to the formation of anti-platelet antibodies and antibody-mediated reticuloendothelial clearance. ${ }^{2}$ Cell-mediated platelet destruction and disruption of $\mathrm{T}$ cell homeostasis are also well described. ${ }^{2-5}$ ITP is also characterized by impaired platelet production secondary to antibody effect in the bone marrow and subsequent megakaryocyte ultrastructural abnormalities. ${ }^{6}$ Primary ITP occurs in the absence of a condition driving immune dysregulation, whereas secondary ITP defines the presence of an associated condition such as hepatitis C, systemic lupus erythematosus, or common variable immunodeficiency. The phase of ITP is classified as "newly diagnosed" in patients less than 3 months from onset, "persistent" in patients from 3 months to 12 months, and "chronic" in patients greater than 12 months from diagnosis. ${ }^{7}$ 
The natural progression of disease varies significantly between pediatric and adult populations. Children typically present with sudden onset of symptoms. Many will experience cutaneous bleeding symptoms including bruising and petechiae. ${ }^{8}$ More severe bleeding ranging from extensive oral bleeding, gastrointestinal bleeding, menorrhagia, or other significant bleeding necessitating urgent medical intervention develops in $20.2 \%$ of patients. $^{9}$ Intracranial hemorrhage (ICH) is estimated to occur in approximately $0.5 \%$ of children with ITP. ${ }^{8,9}$ Spontaneous remission within the first 3 months occurs in approximately $60 \%$, with gradually decreasing frequency thereafter. However, remission continues to occur in those who develop chronic disease. ${ }^{10}$ Because the natural history of disease favors remission and the likelihood of significant bleeding is low, the American Society of Hematology (ASH) Guidelines suggest treatment decisions should be based on bleeding symptoms and other risk factors rather than platelet count. ${ }^{1,11}$ Additionally, the newly published 2019 ASH Guidelines favor outpatient management in children who are asymptomatic or have mild cutaneous bleeding, regardless of platelet count. ${ }^{1}$ Treatment is warranted in children who experience mucosal bleeding and are at risk of developing more severe or life-threatening bleeding. Patients who experience more prolonged periods of thrombocytopenia may require treatment due to healthrelated quality of life (HRQoL) concerns or activity restrictions. First-line therapies in the treatment of pediatric ITP include corticosteroids, intravenous immunoglobulin (IVIG), and anti-D immunoglobulin. These therapies may provide transient benefit and subsequent rise in platelet count, however they have not been shown to impact the duration of immune dysregulation and natural disease course. $^{10}$

ITP is more commonly associated with an indolent course in adults. Up to one-third of patients will be asymptomatic at presentation. ${ }^{12}$ Mucocutaneous bleeding occurs in approximately two-thirds of patients, while 9.6\% experience non-ICH severe bleeding and $1.4 \%$ develop $\mathrm{ICH} .{ }^{9}$ Death from serious bleeding events is estimated to occur in $1-2 \%$ of patients. ${ }^{12,13}$ Spontaneous remission is less common in the adult population with data varying on rate of complete remission. Twenty to $45 \%$ of adults with ITP will experience complete remission within the first 6 months from diagnosis. ${ }^{1,14}$ Spontaneous remission after 6 months is less common than in children but does occur. ${ }^{1}$ Given the predicted course of ITP in adult patients, the goal of therapy is to improve the platelet count to a hemostatic level in order to decrease the risk of serious bleeding. ${ }^{7}$ One to two-thirds of patients achieve this goal with first-line therapy. ${ }^{12}$ In comparison to pediatric patients, the 2019 ASH Guidelines suggest treatment with corticosteroids in adults with a platelet count of less than $30 \times 10^{9} / \mathrm{L}$, regardless of bleeding symptoms at presentation, due to many factors including risk of bleeding, associated comorbidities and/or medications, and increased likelihood of chronic disease. Inpatient management is suggested for newly diagnosed adult patients with severe thrombocytopenia $\left(<20 \times 10^{9} / \mathrm{L}\right)$, regardless of bleeding symptoms due to the importance of diagnostic accuracy in this challenging population. ${ }^{1}$

Patients with ongoing disease and persistent symptoms often require second-line therapies. Additionally, chronic use of first-line therapy places corticosteroid-dependent patients at risk for significant therapy-associated side effects including hypertension, gastric irritation and ulcers, infection, glaucoma, myopathy, osteoporosis, and sleep and mood disturbances. These side effects impact morbidity and further diminish HRQoL. The risk of growth restriction is also concerning in pediatric patients. These and other side effects often necessitate initiation of second-line therapy.

Historically, splenectomy was utilized as the first second-line therapy with $70-80 \%$ of patients achieving an upfront rise in platelet count following surgical intervention. Unfortunately, patients may relapse, and long-term durable remission is achieved in only $50-$ $70 \% .{ }^{15,16}$ Splenectomy within the general population carries the upfront risk of surgical morbidity and mortality as well as the long-term risks of overwhelming infection, thromboembolism, and pulmonary hypertension. ${ }^{17-19}$ These risks have been found to persist beyond 10 years post-splenectomy. ${ }^{19}$ It is unclear whether splenectomy places patients at an increased risk of malignancy as some epidemiological studies have noted an association $^{19-22}$ whereas others have not. ${ }^{21,23}$ Notably, splenectomy rates in patients with ITP have decreased over the last few decades. Medical therapies employed in second-line therapy commonly include rituximab, which aims to suppress antibody-producing $\mathrm{CD} 20+$ B-cells and antibody-mediated clearance of platelets. Rituximab produces a long-term durable response, defined as platelet count $\geq 30 \times 10^{9} / \mathrm{L}$, in approximately $40 \%$ of patients and can be associated with side effects including infusion reactions, infection, and viral reactivation secondary to temporary B-cell aplasia. ${ }^{1}$ Other oral 
immunosuppressive agents have also been utilized with lower efficacy rates. ${ }^{24}$ These therapies also carry the risk of infection, especially when used in combination with other immunosuppressive therapy, as well as individual side effect profiles which should be considered within the context of each patient.

Treatments aimed at increasing platelet production rather than blocking platelet destruction became possible following the discovery of thrombopoietin (TPO) by multiple groups in $1994 .^{25-28}$ With the introduction of thrombopoietin receptor agonists (TPO-RAs), second-line therapy options for ITP patients were expanded to include medications that lacked the significant long-term immunosuppression that characterizes previously available secondline therapies.

\section{Thrombopoietin Biology}

TPO is encoded by the TPO gene located on chromosome $3 \mathrm{q} 27 .{ }^{29}$ It is the largest of the hematopoietic growth factors and consists of two domains: a highly conserved $\mathrm{N}$-terminal domain which is homologous to erythropoietin (EPO) and a C-terminal carbohydrate-rich domain. ${ }^{26}$ Despite the homology with EPO, there is no crossreactivity between the two receptors and EPO cannot activate the TPO receptor, c-mpl. ${ }^{30}$ TPO binds the c-mpl receptor which is encoded by the $M P L$ gene. The c-mpl receptor exists as an inactive homodimer and is activated upon TPO binding. ${ }^{31}$ It is present on hematopoietic stem cells, megakaryocytes, and platelets. ${ }^{30}$

Endogenous TPO, recombinant human TPO, and romiplostim bind inactivated c-mpl at its distal cytokine receptor homology 2 (CRH-2) domain. In contrast, eltrombopag and avatrombopag bind in a transmembrane region of the receptor. ${ }^{30}$ Subsequently, c-mpl is activated and initiates multiple signaling pathways including the JAK-STAT pathway, MAP kinase pathways, as well as anti-apoptotic pathways (Figure 1). ${ }^{30}$ These signaling pathways result in megakaryocyte growth and platelet production.

TPO production and regulation remains incompletely elucidated. TPO production was initially postulated to occur at a constant rate in the liver with regulation of levels via receptor-mediated clearance. ${ }^{30,32}$ TPO bound to c-mpl present on platelets is cleared along with platelets. However, emerging data have demonstrated that TPO regulation is likely more complex than initially postulated. While the liver remains the primary site of production, RNA transcripts have been detected in other tissues including the bone marrow. ${ }^{33}$ Some evidence indicates that platelet granule proteins may directly inhibit TPO mRNA expression and the secretion of TPO in bone marrow stromal cells. ${ }^{34}$ Additionally, clearance of senescent, desialylated platelets via hepatic Ashwell-Morrell receptors appears to increase hepatic TPO production. ${ }^{33}$ In ITP, TPO levels are inappropriately normal with median levels just above those of normal. ${ }^{35}$ It is postulated that in ITP, platelets have increased expression of c-mpl and are thereby able to bind more TPO. ${ }^{36}$ Platelet-bound TPO is then rapidly cleared as platelets are destroyed. ${ }^{30}$

\section{First Generation TPO-RAs: RhTPO and PEG-rHuMGDF}

First-generation TPO-RAs include recombinant human thrombopoietin (rhTPO) and pegylated recombinant human megakaryocyte growth and development factor (PEG-rHuMGDF). ${ }^{37}$ Unfortunately, in 2001 antibodies were identified against PEG-rHuMGDF which also crossreacted with endogenous TPO. In healthy controls, these antibodies resulted in severe thrombocytopenia due to the mitigation of the effects of endogenous TPO. ${ }^{38}$ These antibodies developed due to structural homology between the drug and endogenous TPO and stymied research on recombinant TPO, though various formulations of rhTPO are available in some countries. ${ }^{39,40}$

\section{Second Generation TPO-RAs}

Second-generation TPO-RAs do not share sequence homology with endogenous TPO. The US Food and Drug Administration (FDA) approved second-generation TPO-RAs include romiplostim, a subcutaneous peptideantibody fusion product, as well as eltrombopag and avatrombopag, oral non-peptide small molecules. Romiplostim and eltrombopag are approved for use in children with chronic ITP, and all are approved for use in adults with chronic ITP who have had an insufficient response to first-line therapies.

\section{Molecular Structure and Mechanism of Action of Romiplostim}

Romiplostim is a peptide-antibody fusion product which consists of two dimerized peptides conjugated to the heavy chain of IgG1 (Figure 2). ${ }^{41}$ These peptides are composed of a unique 14 amino acid sequence that lacks sequence homology with endogenous TPO. This decreases immunogenicity and the theoretical risk of subsequent autoantibody formation, greatly reducing the concern that plagued 


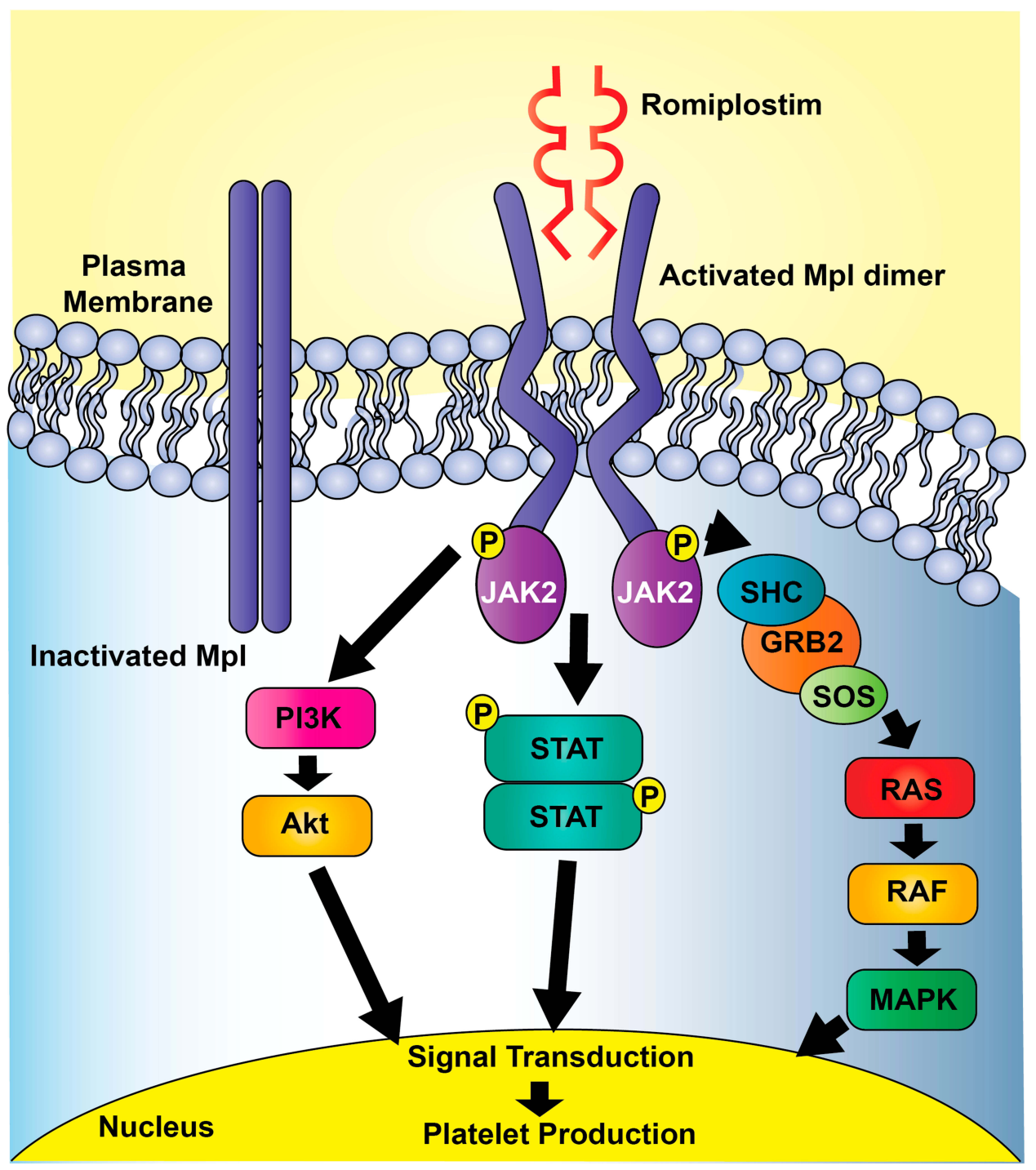

Figure I Activated c-Mpl dimer leads to activation of multiple signaling pathways which promote proliferation and platelet production. These signaling pathways include the SHC-Ras-Raf pathway, JAK-STAT pathway as well as the PI3k-Akt signaling pathway. Figure courtesy of Taylor Olmsted Kim, MD.

Abbreviations: RAF, rapidly accelerated fibrosarcoma; SOS, son of sevenless; GRB2, growth factor receptor-bound protein; JAK2, Janus Kinase 2; STAT, signal transducers and activators of transcription.

first-generation TPO mimetics. ${ }^{30}$ Dimerization of the peptide sequence allows for stimulation of both binding sites located on the TPO receptor. The short circulatory half-life of the dimerized peptide is prolonged by binding to each arm of the IgG1 heavy chain. The resultant "peptibody" is protected from degradation via recycling by endothelial cells through the FcRn receptor. The peptibody is then returned to circulation, extending the half-life of romiplostim to $120-140$ hours. $^{42}$

Romiplostim binds the inactivated c-mpl receptor at the distal CRH-2 domain at the binding site of endogenous TPO. Activation of the c-mpl receptor leads to cell growth via phosphorylation and activation of the JAK and STAT pathways as well as cell maturation via activation of MAPK. ${ }^{30}$ Romiplostim has been shown to increase megakaryocyte size, number, and ploidy resulting in increased platelet production. ${ }^{43}$

\section{Romiplostim Pharmacokinetics and Drug Metabolism}

Romiplostim is given as a subcutaneous injection once weekly. A rise in platelet count generally does not become notable until five days after a dose of romiplostim. Peak platelet counts occur between days 12 and 14 with a decline to baseline by day $28 .{ }^{42}$ There is currently no dose adjustment required for renal or hepatic dysfunction. 


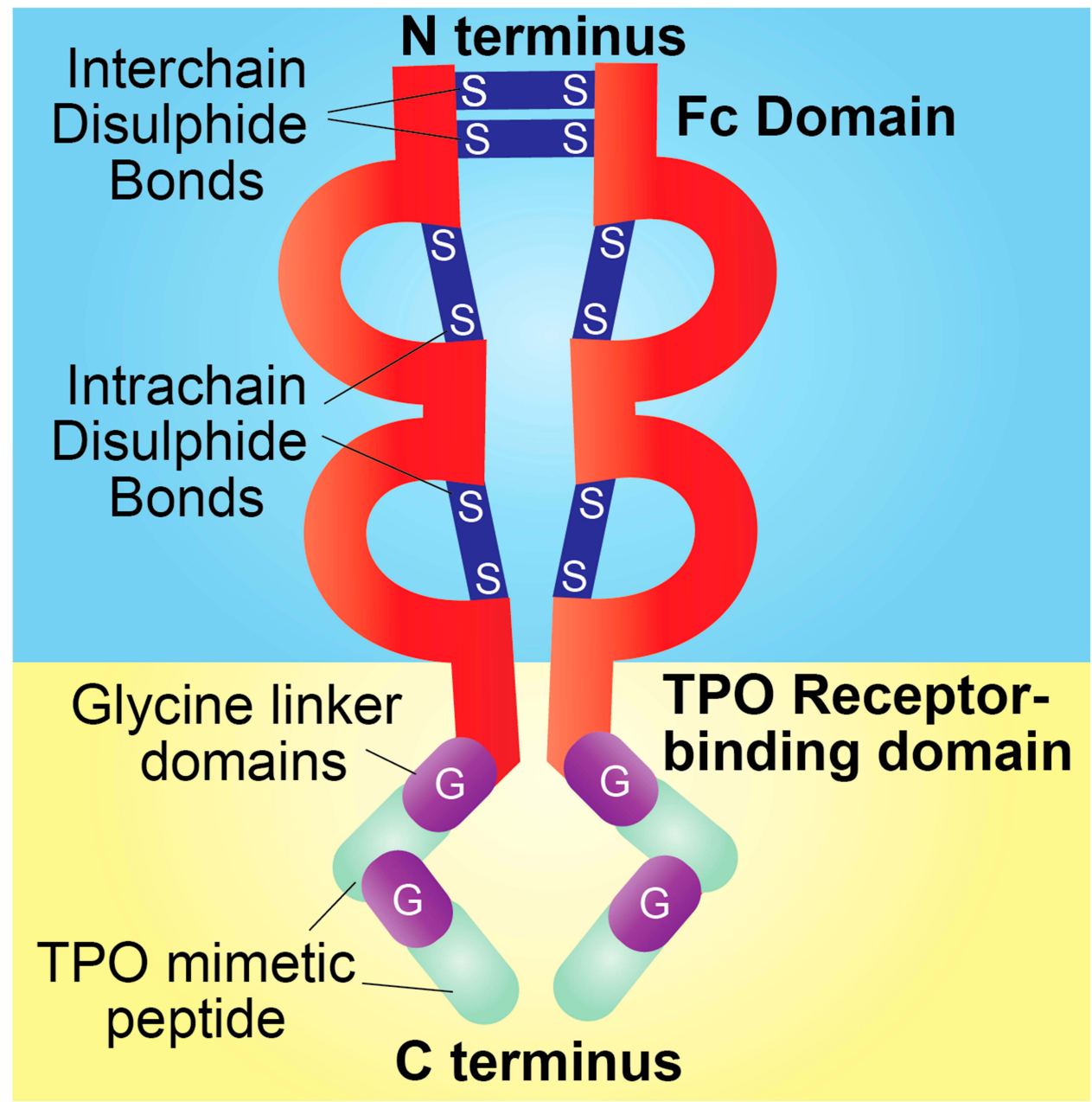

Figure 2 Romiplostim is a recombinant protein comprised of an Fc receptor domain at the $\mathrm{N}$-terminus fused to a thrombopoietin receptor binding domain at the C-terminus. Figure courtesy of Taylor Olmsted Kim, MD.

It is not fully understood to what degree romiplostim undergoes renal filtration, however, at clinically administered doses, the majority of romiplostim is cleared via TPO receptors and reticuloendothelial clearance. ${ }^{44}$ Romiplostim use in pregnancy is discouraged as IgG can cross the placenta via the FcRn receptor. ${ }^{30}$

\section{Current Indications for}

\section{Romiplostim Use in Clinical Practice}

Romiplostim was FDA approved in 2008 for adults and in 2018 for children who have had an inadequate response to first-line therapies in the setting of chronic ITP. The FDA has now expanded approval to adults with newly diagnosed and persistent ITP with insufficient response to firstline therapy or splenectomy. ${ }^{45}$ This expansion was based on data showing median duration of platelet response of 11 months as well as $32 \%$ of patients achieving treatment-free response of at least 6 months' duration following romiplostim administration. ${ }^{46}$ Though only recently FDA approved in children, romiplostim had been used offlabel in pediatric ITP patients for many years prior based on adult data.

Romiplostim induces a reliable platelet response in $>70 \%$ of ITP patients and is associated with decreased bleeding, decreased need for rescue therapies, improvement in patient-reported HRQoL, and an excellent safety profile. ${ }^{47-50}$ Romiplostim can be weaned during the persistent phase of disease to allow for evaluation of sustained remission or if a patient shows evidence of spontaneous recovery. Discontinuation of therapy does not result in longterm complications following resolution of ITP. ${ }^{51}$ However, therapy may also be continued or re-initiated depending on clinical course. Data have shown that response can be maintained with intermittent and prolonged use. ${ }^{52}$ Multiple 
groups have cited treatment-free responses of varying duration and remissions after weaning off TPO-RA therapy. These responses were seen in patients who had previously been refractory to multiple medications. ${ }^{51,53,54}$ The mechanism behind this phenomenon remains unknown.

\section{Safety of Romiplostim in ITP}

Overall, romiplostim is generally safe and well tolerated. Studies have demonstrated a favorable safety profile and low rate of significant treatment-related complications. ${ }^{50}$

\section{Theoretical Risks Associated with Any TPO-RA Therapy}

\section{Thrombosis}

Although studies have confirmed that patients receiving TPO-RA therapy have increased thrombotic risk, the true magnitude of this risk remains uncertain. Potential biases within available data include the baseline increase in thrombotic risk which already exists among patients with ITP, the additive thrombotic risk which prior therapies such as splenectomy may impose, and selection bias in long-term extension cohorts. Based on currently available data, the estimated rate of thromboembolic events is four to five times higher in patients treated with TPO-RAs than in ITP patients unexposed to TPO-RAs, with rates similar between romiplostim and eltrombopag. ${ }^{55}$ Thrombotic events are mostly reported in adults and have been associated with advanced age and co-morbid risk factors such as hypertension, obesity, and smoking. ${ }^{55}$ There were no thromboembolic events reported in children treated with romiplostim in initial industry trials, and no events were reported among a cohort of children treated with romiplostim at Pediatric ITP Consortium of North America (ICON) sites. ${ }^{49,56}$ Thrombotic risk associated with TPO-RA use in patients with ITP does not appear attributable to thrombocytosis and does not demonstrate linear correlation with platelet count. Thrombotic events also do not appear to be consistently related to medication dose. ${ }^{52}$ As the true nature of TPO-RA related thrombotic risk is further elucidated, providers should account for this risk when considering initiation of romiplostim therapy, particularly in older patients and those with comorbidities placing them at higher baseline thrombotic risk.

\section{Rebound Thrombocytopenia}

The risk of rebound thrombocytopenia following abrupt discontinuation of TPO-RA therapy is well documented, and many providers, therefore, wean therapy slowly when discontinuation is planned. The majority of patients with ITP will have platelet counts return to baseline pre-therapy levels once romiplostim is discontinued. However, a small subset of ITP patients may achieve sustained platelet response after romiplostim therapy is weaned. ${ }^{51,53,54}$ Notably, up to $10 \%$ of ITP patients demonstrate marked rebound thrombocytopenia when romiplostim is discontinued, with platelet counts rapidly decreasing below pretreatment levels for 1-3 weeks prior to returning to pretherapy baseline. ${ }^{47}$ One proposed mechanism for this phenomenon is that endogenous TPO activity remains low in the setting of romiplostim therapy due to elevated platelet levels and subsequent TPO clearance via binding to platelet c-mpl receptors. Initially, endogenous TPO activity cannot compensate for the sudden loss of external TPO stimulus following abrupt withdrawal of TPO-RA therapy, resulting in thrombocytopenia below pre-treatment levels. This rebound thrombocytopenia can result in significant bleeding risk. Therefore, experienced providers generally recommend close clinical monitoring and de-escalation of romiplostim dose over abrupt discontinuation of therapy when platelet levels exceed the upper limits of the normal range. Low-dose aspirin may be initiated if platelet count exceeds $800 \times 10^{9} / \mathrm{L}$, although there is no clear evidence that this practice reduces thrombotic risk. These measures aim to prevent clinical bleeding symptoms which may occur in the setting of rebound thrombocytopenia and are likely to pose a higher risk to patients receiving romiplostim therapy than does a transiently elevated platelet count. ${ }^{48}$

\section{Marrow Fibrosis}

Another potential risk associated with the use of TPO-RAs is accelerated bone marrow fibrosis. While reticulin fibers may be seen in the marrow of healthy individuals, collagen fibrosis more definitively signals an underlying pathologic process, most often a myeloproliferative disorder or tumor metastasis. Reticulin fibrosis is more nonspecific and may be associated with a wide range of both benign and pathological conditions. Notably, reticulin fibrosis has been seen in up to one-third of treatmentnaïve ITP patients. ${ }^{57}$ A comprehensive study published in 2013 reported marrow findings among chronic ITP patients receiving long-term romiplostim therapy. ${ }^{52}$ Only $2 \%$ of patients receiving romiplostim for up to 5 years showed evidence of bone marrow fibrosis, with the majority being reticulin fibrosis. Collagen fibrosis was identified in only 1 patient $(0.2 \%$ of all patients $)$ who had preexisting cytogenetic abnormalities known to pre-dispose 
to myelodysplasia. ${ }^{52}$ Bone marrow fibrosis identified in chronic ITP patients receiving romiplostim was not associated with peripheral blood count abnormalities, hypocellularity, or morphological bone marrow abnormalities. Furthermore, reticulin fibrosis appears to be reversible or stable following discontinuation of TPO-RA therapy. Although data is somewhat limited, evidence to date demonstrates no indication for routine bone marrow monitoring in ITP patients receiving TPO-RA therapy. Peripheral blood count and morphology should be monitored routinely, with bone marrow evaluation pursued only in the event of cytopenias or morphological abnormalities.

\section{Myelodysplasia}

There is a theoretical risk of myelodysplasia or clonal evolution via stimulation of c-mpl receptors on hematopoietic stem cells. The potential stimulation of hematopoietic progenitors by TPO-RAs provided the motivation for investigating their utility in aplastic anemia. Reassuringly, a recent study assessing the effects of romiplostim therapy in refractory aplastic anemia showed no evidence of clonal evolution among these patients. ${ }^{58}$ However, further studies are needed to identify and define any potential risks of clonal evolution related to TPO-RA use in the treatment of ITP.

\section{Adverse Effects Associated with Romiplostim Therapy}

Although romiplostim lacks sequence homology with endogenous TPO, it does carry a potential risk for cross-reactive antibody formation, given that it binds at the same domain as endogenous TPO. However, cross-reactive antibody formation to endogenous TPO is exceedingly rare. Only a very small number of patients have been shown to develop neutralizing anti-romiplostim antibodies while receiving romiplostim therapy. These antibodies result in a decrease or loss of platelet response to romiplostim. ${ }^{49,52}$ Cross-reactive neutralizing antibody formation should, therefore, be considered and evaluated clinically when patients with ITP previously responsive to romiplostim develop a sudden decline in or lack of platelet response. Generally, however, the most commonly reported side effects among ITP patients receiving romiplostim are headache, fatigue, and nasopharyngitis. ${ }^{52}$

\section{Decision Factors Surrounding Second-Line Therapy}

Factors that prompt the initiation of second-line therapy in patients with ITP include failure to maintain a sustained response to first-line therapies, bleeding symptoms or risk of bleeding, side effects of first-line therapies, significantly impaired HRQoL, and activity restrictions. Due to a lack of head-to-head prospective comparisons of second-line agents utilized in ITP, there has not been clear guidance regarding the initiation and choice of second-line therapies. Published guidelines have previously not provided a distinct treatment algorithm for management of patients who necessitate second-line therapy which has led to significant variability in institutional preference and physician practice. The 2019 ASH Guidelines include suggestions when comparing second-line therapies that may provide more clinical direction for physicians. ${ }^{1}$ The decision to initiate treatment and which therapy to utilize requires shared decision making given decisions are influenced by numerous factors including patient co-morbidities and clinical status, medication side effect profile, and physician and patient preference.

For patients who have an insufficient response to first-line therapies or who require more long-term treatment, various other options for medical management exist in addition to TPO-RAs. The majority aim to decrease platelet destruction via immunosuppression and include rituximab, mercaptopurine, azathioprine, mycophenolate, and sirolimus, among others. These agents, along with splenectomy, carry the longterm risk of serious infection. Morbidity and mortality secondary to infection is of concern, and the risk-benefit ratio of using these individual therapies must be weighed in light of the relatively low risk of ITP related mortality.

The 2019 ASH Guidelines suggest TPO-RA use in adult patients who are corticosteroid dependent or refractory with duration of disease 3 months or longer, which is a marked change from previous ASH guidelines. ${ }^{1,11}$ Guidelines recommend deferring splenectomy in adults for at least 1 year following diagnosis as some patients may experience spontaneous remission within this period. ${ }^{1}$ TPO-RAs, specifically romiplostim in light of its expanded FDA approval in acute and persistent ITP, may provide benefit as a medical bridge to patients who are eligible for and prefer surgery.

\section{ICONI: Pediatric Treatment Outcomes and Drivers of Physician Decision Making} The Pediatric ITP Consortium of North America (ICON) sought to better describe the use of second-line therapies in pediatric ITP patients with a prospective longitudinal observational cohort study of patients initiating secondline therapies, ICON1. ${ }^{24,59}$ Data demonstrate that while TPO-RAs are approved for pediatric patients with chronic 
ITP, physicians are utilizing these agents earlier in the course of disease as well. ${ }^{24}$ In this study, $17 \%$ of patients received second-line therapy within the first three months and another $30 \%$ of patients received second-line therapy between 3 and 12 months. Due to the lack of head-to-head trials comparing second-line therapies, the study sought to provide a comparison of treatment outcomes including platelet count, bleeding symptoms, and HRQoL at baseline as well as 1, 6, and 12 months after initiation of therapy. Due to attrition in all groups, data for primary aims were compared up to 6 months. Patients receiving oral immunosuppressants had the highest rate of study withdrawal at 12 months due to lack of treatment response or side effects. Romiplostim had the highest rate of complete platelet response (CR) or partial platelet response (PR) at 6 months of therapy (CR 71\%, PR 15\%) when compared with eltrombopag (CR 27\%, PR 40\%), rituximab (CR $52 \%$, PR 27\%), and oral immunosuppressants (CR 25\%, PR 13\%). The majority of patients receiving romiplostim who were classified as non-responders at 1 month went on to achieve complete or partial response at 6 months. Platelet response to oral immunosuppressants was half that of other second-line therapies. Bleeding symptoms improved with all second-line therapies with the most significant reduction occurring in the rituximab and romiplostim groups. Patients receiving romiplostim and rituximab required significantly less rescue therapy $(12.5 \%$ and $6.1 \%$, respectively). Notably, HRQoL improved in patients across all treatments but was most pronounced in patients receiving rituximab and eltrombopag at 12 months of therapy. ${ }^{24}$ Based on this data, it was postulated that various agents may be selected depending on the end point physicians and patients wish to achieve. Romiplostim may be most beneficial in patients who experience recurrent bleeding given its high rates of $\mathrm{CR}$ and PR. Rituximab may also be a beneficial choice for this same reason or in patients who are experiencing significant HRQoL concerns.

ICON1 also sought to better determine drivers of physician decision making when selecting second-line therapies. Data indicate the main drivers of physician decision include patient and family preference as well as medication-specific factors including side effect profile, possibility of long-term remission, ease of use, and long-term toxicity (Figure 3). ${ }^{59}$ In pediatric patients with chronic ITP, patient and family preference is the most significant influencing factor in a physician's choice of therapy. Less than $10 \%$ of physicians cite published guidelines as a factor contributing to their therapy choice. Physicians are more likely to choose romiplostim over other agents due to expected efficacy as well as its overall side effect profile. Eltrombopag and oral immunosuppressive agents are more often selected for their ease of administration while splenectomy and rituximab are chosen due to the possibility of long-term remission. ${ }^{59}$

\section{American Society of Hematology (ASH) Guidelines 2019}

Due to the paucity of head-to-head prospective comparisons of the many ITP therapies, particularly those utilized as second-line agents, the ASH Guidelines panel sought to prioritize treatment strategies that avoided significant medication-related side effects. Observation continues to be the suggested route of management in newly diagnosed pediatric patients who are asymptomatic or have only mild cutaneous bleeding, irrespective of platelet counts. TPORAs are now included in second-line therapy, and their use in children who fail to respond to first-line therapy is suggested over rituximab and splenectomy. ${ }^{1}$ In adult patients with ITP of 3 months' duration or longer who are corticosteroid dependent or unresponsive, TPO-RAs are suggested over rituximab, and rituximab is suggested over splenectomy. The guidelines highlight the importance of shared decision making with a focus on patient preferences and patient-specific factors including duration of disease, frequency of bleeding, need for rescue therapies, and comorbidities. Additional factors influencing treatment decisions include medication cost and availability, as well as psychosocial factors such as available support systems and the impact of disease and treatments on HRQoL. ${ }^{1}$

\section{Choosing Between Second Generation TPO-RAs}

The 2019 ASH Guidelines recommend eltrombopag or romiplostim with emphasis placed on individual patient preference. No difference was found between these two TPO-RAs in regards to rate of durable response, overall bleeding, clinically significant bleeding, need for rescue therapy, and adverse events by meta-analysis. ${ }^{60}$ Avatrombopag was FDA approved in June 2019 for use in adult patients with chronic ITP who have failed to respond to previous therapy. ${ }^{61}$ Due to its recent approval, it is not specifically addressed in the most recent guidelines. Table 1 compares these three medications, focusing on factors both patients and physicians should consider 
Parental or Patient Preference

Physician Comfort Ease of Administration

Most Efficacious

Side Effect Profile

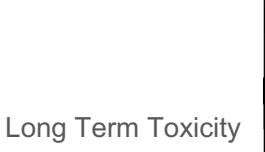

$$
\begin{array}{|}
\hline \\
\hline \\
\hline \\
\hline \\
\hline
\end{array}
$$

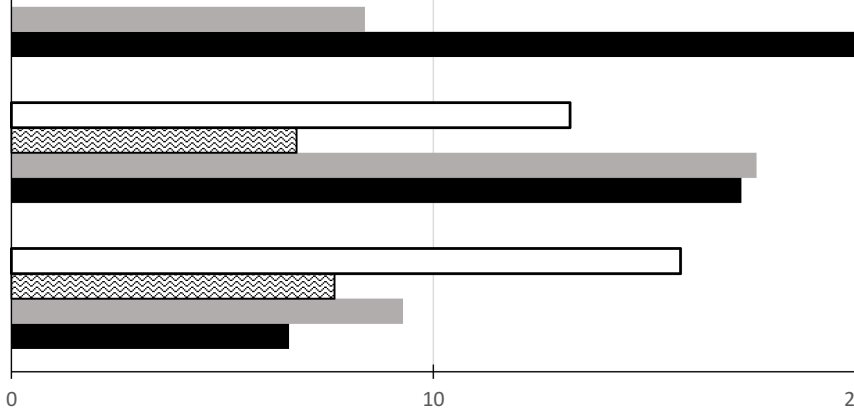

Ranked Score

口Oral Immunosuppressants

QRituximab

Eltrombopag

- Romiplostim

Figure 3 Selected factors affecting physician choice of second-line therapy at ICON treatment sites. Weighted scores were assigned to reasons for physician choice within individual therapies. Equal length bars indicate the reason was equally ranked for each treatment. Adapted with permission from Grace RF, Despotovic JM, Bennett CM, et al. Physician decision making in selection of second-line treatments in immune thrombocytopenia in children. Am J Hematol. 20I8;93(7):882-888e. (C) 20I8 Wiley Periodicals, Inc. ${ }^{59}$

when choosing between TPO-RAs. Each medication has varying insurance coverage which impacts patient access to therapy. Historically, subcutaneous romiplostim injections have required weekly clinic visits and patients incur time and monetary costs associated with these medical visits. Currently, romiplostim does not have universal approval for self-administration at home; however, selfadministration is available in some countries.

While the decision for type and timing of second-line therapy, including selection of preferred TPO-RA, should

Table I Comparing TPO-RAs

\begin{tabular}{|c|c|c|c|c|}
\hline Medication & Route & Frequency & Special Considerations & $\begin{array}{l}\text { Average Wholesale Price for } 30 \\
\text { Days }^{\text {a }}\end{array}$ \\
\hline Romiplostim & Subcutaneous & $\begin{array}{l}\text { Once } \\
\text { weekly }\end{array}$ & $\begin{array}{l}\text { - Weekly medical visits for dose titration } \\
\text { - Lack of universal availability for home administration }\end{array}$ & $\begin{array}{l}\$ 4460 \text { to } \$ 17,840 \text { depending on vial } \\
\text { size }\end{array}$ \\
\hline Eltrombopag & Oral & Once daily & $\begin{array}{l}\text { - Powder preparation for reconstitution recently } \\
\text { available } \\
\text { - Frequent lab visits during dose titration } \\
\text { - Significant dietary restrictions due to interaction } \\
\text { with divalent cations } \\
\text { - Potential for iron chelation } \\
\text { - Hepatotoxicity }\end{array}$ & $\begin{array}{l}\$ 5910 \text { to } \$ 32,095 \text { depending on tablet } \\
\text { strength }\end{array}$ \\
\hline Avatrombopag & Oral & Once daily & $\begin{array}{l}\text { - No liquid preparation or powder for reconstitution } \\
\text { available } \\
\text { - Frequent lab visits during dose titration } \\
\text { - No dietary restrictions } \\
\text { - No data available in children }\end{array}$ & $\begin{array}{l}\$ 1425 \text { to } \$ 21,384 \text { depending on dose } \\
\text { and frequency }\end{array}$ \\
\hline
\end{tabular}

Note: ${ }^{a}$ Average wholesale price (AWP) as reported on Lexicomp. ${ }^{62}$ 
be based on clinical factors including platelet count, bleeding symptoms, and associated comorbidities, selected treatment should also be aimed at improving HRQoL for each individual patient. Theoretically, successful treatment with a therapy associated with minimal side effects and patient imposition would lead to improvement in HRQoL for both patients and their caregivers.

\section{Health-Related Quality of Life}

Medical decisions regarding choice and initiation of second-line therapy in ITP are made on a case-by-case basis and consider physician and patient preferences. In addition to minimizing treatment-related side effects and maximizing treatment response, therapy decisions should also aim to minimize the negative impact on quality of life (QoL) for patients and caregivers. HRQoL is negatively impacted by ITP. $^{63}$ Adults with ITP demonstrate lower HRQoL scores than the general population, comparable to patients with other chronic diseases. ${ }^{64}$ Children affected by ITP report lower HRQoL scores compared to peers. Additionally, substantial caregiver strain is well documented. $^{63,65}$ Generic HRQoL tools were initially employed; however, ITP specific HRQoL tools have been developed and validated to better explore diseasespecific symptoms and issues. HRQoL tools are specific to adult or pediatric populations.

\section{Health-Related Quality of Life: Children}

HRQoL improves for both children and caregivers after 12 months of disease, reflective of the natural progression of pediatric disease with most spontaneously resolving by 12 months. ${ }^{63,65}$ Interestingly, improvement in HRQoL scores also occurs in chronic patients after 12 months, compared to HRQoL scores shortly after diagnosis. ${ }^{63}$ The Kids' ITP Tool (KIT) was developed to further elucidate the impact of disease burden on patients and caregivers and has been shown to be valid and reliable. ${ }^{66}$ KIT involves three sections: a child self-report version for children 7 years of age and older, a parent-proxy version completed by caregivers for children 6 years and younger, and a parental impact version. As HRQoL began to be more systematically studied in children, KIT questionnaires were incorporated into clinical trials of ITP therapies.

There have been two studies conducted on the safety, tolerability, and efficacy of romiplostim in pediatric ITP patients which incorporated sequential KIT evaluations. ${ }^{67,68}$ In both the pilot study and Phase 3 trial, there were no statistically significant changes in child-self report scores, signifying romiplostim therapy did not significantly alter patients' perceived HRQoL. However, in both studies individually, there was some evidence of improved parental impact scores in those treated with romiplostim versus controls, signifying possible decrease in parental burden. ${ }^{69}$

Parental fear of bleeding significantly impacts HRQoL for caregivers with parental impact scores being lower than child-report score. ${ }^{65,69}$ The reduction of parental burden in the setting of a high response rate to romiplostim supports the idea that parental burden and worry are likely dependent on platelet count and therapy response. Conversely, the lack of improvement in self-report and parent-proxy scores suggests other factors such as activity limitations, medical visits, and anxiety with venipuncture likely drive decreased HRQoL scores among children. ${ }^{63}$

\section{Patient and Parent Preference: Insight from Physician Experience}

Data regarding factors influencing patient or parent preference and decision making is lacking. In these authors' experience, patient or parent preferences vary considerably, but most commonly prioritize availability of medication, ease of achieving optimal adherence, and side effect profile. Patients or parents may feel adherence is better achieved with weekly subcutaneous dosing of romiplostim when compared to daily oral medications. Eltrombopag requires dietary modification around the time of medication administration due to the presence of divalent cations such as calcium, magnesium and iron, which interfere with medication absorption. These dietary restrictions may be limiting, particularly in young children. Others prefer taking an oral medication (eltrombopag, avatrombopag) over a subcutaneous injection (romiplostim). This may be particularly relevant when home administration of romiplostim is not possible. The side effect profiles among TPO-RAs are similar with the exception of transaminitis associated with eltrombopag. Parents or patients sometimes cite this difference as the reason for their choice of romiplostim over eltrombopag. Efficacy is similar among TPO-RAs but may vary for each individual, thus influencing a patient's ultimate choice of TPORA. ${ }^{49,50,61,70}$ Failure to respond to one TPO-RA does not preclude response to another. ${ }^{71}$ When selecting second-line therapy, tailoring each patient's treatment to his or her individual preference and goals 
of care may lead to increased HRQoL at the individual level.

\section{Health-Related Quality of Life: Adults}

In adults, HRQoL deteriorates in the first year with subsequent improvement $>12$ months following diagnosis. ${ }^{72}$ Patients requiring treatment for ITP consistently have lower HRQoL scores which may reflect overall severity of disease, impact of treatment on HRQoL, or both. ${ }^{64}$ Overall a patient's individual HRQoL scores increase with treatment of ITP. ${ }^{72,73}$

One of the most frequently utilized ITP specific assessment tools for adults, the Immune Thrombocytopenia Purpura Patient Assessment Questionnaire (ITP-PAQ), demonstrates responsiveness, reliability, and validity in the majority of domains assessed. ${ }^{74}$ The tool assesses physical health, evaluating symptoms including bleeding, bother, fatigue and activity, as well as emotional health, tabulating fear and psychological health. The ITP-PAQ also evaluates the impact of ITP and platelet-related therapies on social activity, work, and overall QoL. An additional domain exists for further evaluation of women's reproductive health which is comprised of menstrual symptoms and fertility sub-scales. ${ }^{74}$

In pooled data from two placebo-controlled phase 3 trials of romiplostim in adults with chronic ITP, patients receiving romiplostim demonstrated a significant improvement in scores in seven of the ten scales assessed by ITPPAQ. These included improved scores in symptoms, bother, activity, fear, psychological health, social activity, and women's reproductive health (menstrual symptoms sub-scale only). No significant differences between those treated with romiplostim versus placebo were noted in the remaining scales, notably overall QoL and fatigue. ${ }^{73}$

Similarly, in a randomized open label 52-week trial of romiplostim versus standard of care (SOC) in adults with ITP refractory to prior therapy, those receiving romiplostim demonstrated statistically significant improvements in HRQoL scores in seven scales including symptoms, bother, activity, psychological health, fear, social QoL, and overall QoL. Again, no improvement in fatigue scale scores was noted for either treatment group. While patients treated with romiplostim demonstrated statistically significant increases in HRQoL scores on multiple scales when compared to patients receiving SOC, the clinical impact represented by this difference is difficult to determine and may not be noticeable to patients. $^{72}$

\section{Conclusion}

Romiplostim, along with other TPO-RAs, is safe and efficacious in the treatment of adults and children with ITP refractory to first-line therapies. Because of this, the 2019 ASH ITP Guidelines suggest the use of TPO-RAs over other previously employed second-line therapies. Shared decision making between physicians, patients, and families is critical and should consider laboratory data, bleeding symptoms, comorbidities, patient preference, and HRQoL concerns. Romiplostim is well tolerated, however some aspects of the weekly subcutaneous injection may not be acceptable for some patients. Data suggest romiplostim may be most beneficial in patients with persistent bleeding symptoms given the high rate of platelet response. Romiplostim therapy improves HRQoL from baseline; however, each individual patient may experience varying improvement due to individual goals of therapy and response to platelet-directed therapy.

\section{Author Contributions}

All authors contributed to data analysis, drafting or revising the article, gave final approval of the version to be published, and agree to be accountable for all aspects of the work.

\section{Disclosure}

JMD has received research funding from Amgen and Novartis, and has served as a Consultant for Dova Pharmaceuticals. JMD also reports personal fees from Dova Pharmaceuticals, grants, personal fees from Novartis, grants from Amgen, personal fees from SanofiGenzyme, outside the submitted work. The authors report no other conflicts of interest in this work.

\section{References}

1. Neunert C, Terrell DR, Arnold DM, et al. American society of hematology 2019 guidelines for immune thrombocytopenia. Blood $A d v$. 2019;3(23):3829-3866. doi:10.1182/bloodadvances.2019000966

2. Zufferey A, Kapur R, Semple JW. Pathogenesis and therapeutic mechanisms in immune thrombocytopenia (ITP). J Clin Med. 2017;6 (2): 16 . doi: $10.3390 / \mathrm{jcm} 6020016$

3. Yazdanbakhsh K. Imbalanced immune homeostasis in immune thrombocytopenia. Semin Hematol. 2016;53(Suppl 1):S16-S19. doi:10.1053/j.seminhematol.2016.04.006

4. Olsson B, Andersson PO, Jernas M, et al. T-cell-mediated cytotoxicity toward platelets in chronic idiopathic thrombocytopenic purpura. Nat Med. 2003;9(9):1123-1124. doi:10.1038/nm921

5. Liu B, Zhao H, Poon MC, et al. Abnormality of CD4(+)CD25(+) regulatory $\mathrm{T}$ cells in idiopathic thrombocytopenic purpura. Eur $J$ Haematol. 2007;78(2):139-143. doi:10.1111/j.1600-0609.2006.00780.x

6. Houwerzijl EJ, Blom NR, van der Want JJ, et al. Ultrastructural study shows morphologic features of apoptosis and para-apoptosis in megakaryocytes from patients with idiopathic thrombocytopenic purpura. Blood. 2004;103(2):500-506. doi:10.1182/blood-2003-01-0275 
7. Rodeghiero F, Stasi R, Gernsheimer T, et al. Standardization of terminology, definitions and outcome criteria in immune thrombocytopenic purpura of adults and children: report from an international working group. Blood. 2009;113(11):2386-2393. doi:10.1182/blood2008-07-162503

8. Kuhne T, Berchtold W, Michaels LA, et al. Newly diagnosed immune thrombocytopenia in children and adults: a comparative prospective observational registry of the intercontinental cooperative immune thrombocytopenia study group. Haematologica. 2011;96 (12):1831-1837. doi:10.3324/haematol.2011.050799

9. Neunert C, Noroozi N, Norman G, et al. Severe bleeding events in adults and children with primary immune thrombocytopenia: a systematic review. J Thromb Haemost. 2015;13(3):457-464. doi: $10.1111 /$ jth. 12813

10. Heitink-Polle KMJ, Uiterwaal C, Porcelijn L, et al. Intravenous immunoglobulin vs observation in childhood immune thrombocytopenia: a randomized controlled trial. Blood. 2018;132(9):883-891. doi:10.1182/blood-2018-02-830844

11. Neunert C, Lim W, Crowther M, et al. The American Society of Hematology 2011 evidence-based practice guideline for immune thrombocytopenia. Blood. 2011;117(16):4190-4207. doi:10.1182/ blood-2010-08-302984

12. Neylon AJ, Saunders PW, Howard MR, Proctor SJ, Taylor PR. Northern Region Haematology G. Clinically significant newly presenting autoimmune thrombocytopenic purpura in adults: a prospective study of a population-based cohort of 245 patients. $\mathrm{Br}$ $J$ Haematol. 2003;122(6):966-974. doi:10.1046/j.1365-2141.20 03.04547.x

13. Portielje JE, Westendorp RG, Kluin-Nelemans HC, Brand A. Morbidity and mortality in adults with idiopathic thrombocytopenic purpura. Blood. 2001;97(9):2549-2554. doi:10.1182/blood. V97.9.2549

14. Schifferli A, Holbro A, Chitlur M, et al. A comparative prospective observational study of children and adults with immune thrombocytopenia: 2-year follow-up. Am J Hematol. 2018;93(6):751-759. doi:10.1002/ajh.25086

15. Ahmed R, Devasia AJ, Viswabandya A, et al. Long-term outcome following splenectomy for chronic and persistent immune thrombocytopenia (ITP) in adults and children: splenectomy in ITP. Ann Hematol. 2016;95(9):1429-1434. doi:10.1007/s00277-016-2738-3

16. Mazzucconi MG, Arista MC, Peraino M, et al. Long-term follow-up of autoimmune thrombocytopenic purpura (ATP) patients submitted to splenectomy. Eur J Haematol. 1999;62(4):219-222. doi:10.1111/ j.1600-0609.1999.tb01750.x

17. Ejstrud P, Kristensen B, Hansen JB, Madsen KM, Schonheyder HC, Sorensen HT. Risk and patterns of bacteraemia after splenectomy: a population-based study. Scand J Infect Dis. 2000;32(5):521-525. doi: $10.1080 / 003655400458811$

18. Theilacker C, Ludewig K, Serr A, et al. Overwhelming postsplenectomy infection: a prospective multicenter cohort study. Clin Infect Dis. 2016;62(7):871-878. doi:10.1093/cid/civ1195

19. Kristinsson SY, Gridley G, Hoover RN, Check D, Landgren O. Longterm risks after splenectomy among 8149 cancer-free American veterans: a cohort study with up to 27 years follow-up. Haematologica. 2014;99(2):392-398. doi:10.3324/haematol.2013.092460

20. Linet MS, Nyren O, Gridley G, et al. Causes of death among patients surviving at least one year following splenectomy. Am J Surg. 1996;172(4):320-323. doi:10.1016/S0002-9610(96)00196-1

21. Mellemkjoer L, Olsen JH, Linet MS, Gridley G, McLaughlin JK. Cancer risk after splenectomy. Cancer. 1995;75(2):577-583. doi:10.1002/10970142(19950115)75:2<577::AID-CNCR2820750222>3.0.CO;2-K

22. Sun LM, Chen HJ, Jeng LB, Li TC, Wu SC, Kao CH. Splenectomy and increased subsequent cancer risk: a nationwide population-based cohort study. Am J Surg. 2015;210(2):243-251. doi:10.1016/j. amjsurg.2015.01.017
23. Robinette CD, Fraumeni JF Jr. Splenectomy and subsequent mortality in veterans of the 1939-45 war. Lancet. 1977;2(8029):127-129. doi:10.1016/S0140-6736(77)90132-5

24. Grace RF, Shimano KA, Bhat R, et al. Second-line treatments in children with immune thrombocytopenia: effect on platelet count and patient-centered outcomes. Am J Hematol. 2019;94(7):741-750. doi:10.1002/ajh.25479

25. Bartley TD, Bogenberger J, Hunt P, et al. Identification and cloning of a megakaryocyte growth and development factor that is a ligand for the cytokine receptor Mpl. Cell. 1994;77(7):1117-1124. doi:10.1016/0092-8674(94)90450-2

26. de Sauvage FJ, Hass PE, Spencer SD, et al. Stimulation of megakaryocytopoiesis and thrombopoiesis by the c-Mpl ligand. Nature. 1994;369(6481):533-538. doi:10.1038/369533a0

27. Kuter DJ, Beeler DL, Rosenberg RD. The purification of megapoietin: a physiological regulator of megakaryocyte growth and platelet production. Proc Natl Acad Sci U S A. 1994;91(23):11104-11108. doi:10.1073/pnas.91.23.11104

28. Lok S, Kaushansky K, Holly RD, et al. Cloning and expression of murine thrombopoietin cDNA and stimulation of platelet production in vivo. Nature. 1994;369(6481):565-568. doi:10.1038/369565a0

29. Foster DC, Sprecher CA, Grant FJ, et al. Human thrombopoietin: gene structure, cDNA sequence, expression, and chromosomal localization. Proc Natl Acad Sci U S A. 1994;91(26):13023-13027. doi:10.1073/pnas.91.26.13023

30. Kuter DJ. The biology of thrombopoietin and thrombopoietin receptor agonists. Int J Hematol. 2013;98(1):10-23. doi:10.1007/s12185-0131382-0

31. Kim TO, Despotovic J, Lambert MP. Eltrombopag for use in children with immune thrombocytopenia. Blood Adv. 2018;2(4):454-461. doi:10.1182/bloodadvances.2017010660

32. Li J, Xia Y, Kuter DJ. Interaction of thrombopoietin with the platelet c-mpl receptor in plasma: binding, internalization, stability and pharmacokinetics. $\quad B r \quad J \quad$ Haematol. 1999;106(2):345-356. doi:10.1046/j.1365-2141.1999.01571.x

33. Grozovsky R, Giannini S, Falet H, Hoffmeister KM. Novel mechanisms of platelet clearance and thrombopoietin regulation. Curr Opin Hematol. 2015;22(5):445-451. doi:10.1097/MOH.0000000000000170

34. McIntosh B, Kaushansky K. Transcriptional regulation of bone marrow thrombopoietin by platelet proteins. Exp Hematol. 2008;36 (7):799-806. doi:10.1016/j.exphem.2008.02.012

35. Kuter DJ, Gernsheimer TB. Thrombopoietin and platelet production in chronic immune thrombocytopenia. Hematol Oncol Clin North Am. 2009;23(6):1193-1211. doi:10.1016/j.hoc.2009.09.001

36. Nichol JL, Endogenous TPO (eTPO) levels in health and disease: possible clues for therapeutic intervention. Stem Cells. 1998;16(Suppl 2):165-175. doi:10.1002/stem.5530160719

37. Kuter DJ. Whatever happened to thrombopoietin? Transfusion. Mar. 2002;42(3):279-283.

38. Li J, Yang C, Xia Y, et al. Thrombocytopenia caused by the development of antibodies to thrombopoietin. Blood. 2001;98 (12):3241-3248. doi:10.1182/blood.V98.12.3241

39. Kong Z, Qin P, Xiao S, et al. A novel recombinant human thrombopoietin therapy for the management of immune thrombocytopenia in pregnancy. Blood. 2017;130(9):1097-1103. doi:10.1182/blood-2017-01-761262

40. Wang S, Yang R, Zou P, et al. A multicenter randomized controlled trial of recombinant human thrombopoietin treatment in patients with primary immune thrombocytopenia. Int $J$ Hematol. 2012;96 (2):222-228. doi:10.1007/s12185-012-1124-8

41. Molineux G. The development of romiplostim for patients with immune thrombocytopenia. Ann N Y Acad Sci. 2011;1222(1):55-63. doi:10.1111/j.1749-6632.2011.05975.x

42. Wang B, Nichol JL, Sullivan JT. Pharmacodynamics and pharmacokinetics of AMG 531, a novel thrombopoietin receptor ligand. Clin Pharmacol Ther. 2004;76(6):628-638. doi:10.1016/j. clpt.2004.08.010 
43. Broudy VC, Lin NL. AMG531 stimulates megakaryopoiesis in vitro by binding to Mpl. Cytokine. 2004;25(2):52-60. doi:10.1016/j. cyto.2003.05.001

44. Wang YM, Krzyzanski W, Doshi S, Xiao JJ, Perez-Ruixo JJ, Chow AT. Pharmacodynamics-mediated drug disposition (PDMDD) and precursor pool lifespan model for single dose of romiplostim in healthy subjects. AAPS J. 2010;12(4):729-740. doi:10.1208/s12248010-9234-9

45. Romiplostim. 2019; Available from: https://www.accessdata.fda.gov/ drugsatfda_docs/label/2019/125268s164lbl.pdf. Accessed November 20,2019

46. Newland A, Godeau B, Priego V, et al. Remission and platelet responses with romiplostim in primary immune thrombocytopenia: final results from a Phase 2 study. $\mathrm{Br} J$ Haematol. 2016;172 (2):262-273. doi:10.1111/bjh.13827

47. Kuter DJ, Bussel JB, Lyons RM, et al. Efficacy of romiplostim in patients with chronic immune thrombocytopenic purpura: a double-blind randomised controlled trial. Lancet. 2008;371 (9610):395-403. doi:10.1016/S0140-6736(08)60203-2

48. Kuter DJ, Rummel M, Boccia R, et al. Romiplostim or standard of care in patients with immune thrombocytopenia. $N$ Engl J Med. 2010;363(20):1889-1899. doi:10.1056/NEJMoa1002625

49. Neunert C, Despotovic J, Haley K, et al. Thrombopoietin receptor agonist use in children: data from the pediatric ITP consortium of north america ICON2 study. Pediatr Blood Cancer. 2016;63 (8):1407-1413. doi:10.1002/pbc.26003

50. Cines DB, Wasser J, Rodeghiero F, et al. Safety and efficacy of romiplostim in splenectomized and nonsplenectomized patients with primary immune thrombocytopenia. Haematologica. 2017;102 (8):1342-1351. doi:10.3324/haematol.2016.161968

51. Ghadaki B, Nazi I, Kelton JG, Arnold DM. Sustained remissions of immune thrombocytopenia associated with the use of thrombopoietin receptor agonists. Transfusion. 2013;53(11):2807-2812. doi:10.1111/ trf. 12139

52. Rodeghiero F, Stasi R, Giagounidis A, et al. Long-term safety and tolerability of romiplostim in patients with primary immune thrombocytopenia: a pooled analysis of 13 clinical trials. Eur J Haematol. 2013;91(5):423-436. doi:10.1111/ejh.12181

53. Cervinek L, Mayer J, Doubek M. Sustained remission of chronic immune thrombocytopenia after discontinuation of treatment with thrombopoietin-receptor agonists in adults. Int $J$ Hematol. 2015;102 (1):7-11. doi:10.1007/s12185-015-1793-1

54. Mahevas M, Fain O, Ebbo M, et al. The temporary use of thrombopoietin-receptor agonists may induce a prolonged remission in adult chronic immune thrombocytopenia. Results of a French observational study. Br J Haematol. 2014;165(6):865-869. doi:10.1111/bjh.12888

55. Rodeghiero F. Is ITP a thrombophilic disorder? Am J Hematol. Jan. 2016;91(1):39-45

56. Tarantino MD, Bussel JB, Blanchette VS, et al. Romiplostim in children with immune thrombocytopenia: a phase 3, randomised, double-blind, placebo-controlled study. Lancet. 2016;388 (10039):45-54. doi:10.1016/S0140-6736(16)00279-8

57. Rizvi H, Butler T, Calaminici M, et al. United Kingdom immune thrombocytopenia registry: retrospective evaluation of bone marrow fibrosis in adult patients with primary immune thrombocytopenia and correlation with clinical findings. $B r \quad J$ Haematol. 2015;169 (4):590-594. doi:10.1111/bjh.13330

58. Lee JW, Lee SE, Jung CW, et al. Romiplostim in patients with refractory aplastic anaemia previously treated with immunosuppressive therapy: a dose-finding and long-term treatment phase 2 trial. Lancet Haematol. 2019;6(11):e562-e572. doi:10.1016/S2352-3026(19)30153-X

59. Grace RF, Despotovic JM, Bennett CM, et al. Physician decision making in selection of second-line treatments in immune thrombocytopenia in children. Am $J$ Hematol. 2018;93(7):882-888. doi:10.1002/ajh.25110
60. Zhang J, Liang Y, Ai Y, et al. Eltrombopag versus romiplostim in treatment of adult patients with immune thrombocytopenia: a systematic review incorporating an indirect-comparison meta-analysis. PLoS One. 2018;13(6):e0198504. doi:10.1371/journal.pone.0198504

61. Jurczak W, Chojnowski K, Mayer J, et al. Phase 3 randomised study of avatrombopag, a novel thrombopoietin receptor agonist for the treatment of chronic immune thrombocytopenia. $\mathrm{Br} J$ Haematol. 2018;183(3):479-490. doi:10.1111/bjh.15573

62. Lexicomp Online PaNL-DO, Hudson, Ohio: wolters Kluwer Clinical Drug Information, Inc. December. 2019;6:2019.

63. Trotter P, Hill QA. Immune thrombocytopenia: improving quality of life and patient outcomes. Patient Relat Outcome Meas. 2018;9:369-384. doi:10.2147/PROM.S140932

64. McMillan R, Bussel JB, George JN, Lalla D, Nichol JL. Self-reported health-related quality of life in adults with chronic immune thrombocytopenic purpura. Am J Hematol. 2008;83(2):150-154. doi:10.1002/ ajh.20992

65. Flores A, Klaassen RJ, Buchanan GR, Neunert CE. Patterns and influences in health-related quality of life in children with immune thrombocytopenia: a study from the Dallas ITP Cohort. Pediatr Blood Cancer. 2017;64(8):e26405. doi:10.1002/pbc.26405

66. Klaassen RJ, Blanchette VS, Barnard D, et al. Validity, reliability, and responsiveness of a new measure of health-related quality of life in children with immune thrombocytopenic purpura: the Kids' ITP Tools. J Pediatr. 2007;150(5):510-515. doi:10.1016/j.jpeds.2007.01.037

67. Klaassen RJ, Mathias SD, Buchanan G, et al. Pilot study of the effect of romiplostim on child health-related quality of life (HRQoL) and parental burden in immune thrombocytopenia (ITP). Pediatr Blood Cancer. 2012;58(3):395-398. doi:10.1002/pbc.23312

68. Mathias SD, Li X, Eisen M, Carpenter N, Crosby RD, Blanchette VS. A phase 3, randomized, double-blind, placebo-controlled study to determine the effect of romiplostim on health-related quality of life in children with primary immune thrombocytopenia and associated burden in their parents. Pediatr Blood Cancer. 2016;63 (7):1232-1237. doi:10.1002/pbc.25984

69. Guo JC, Zheng Y, Chen HT, et al. Efficacy and safety of thrombopoietin receptor agonists in children with chronic immune thrombocytopenia: a meta-analysis. Oncotarget. 2018;9(6):7112-7125. doi:10.18632/oncotarget.23487

70. Wong RSM, Saleh MN, Khelif A, et al. Safety and efficacy of long-term treatment of chronic/persistent ITP with eltrombopag: final results of the EXTEND study. Blood. 2017;130 (23):2527-2536. doi:10.1002/ajh.23163

71. Gonzalez KJ, Zuluaga SO, DaRos CV, Rodriguez PP, Marti AC. Sequential treatment with thrombopoietin-receptor agonists (TPO-RAs) in immune thrombocytopenia (ITP): experience in our center. Ann Hematol. 2017;96(3):507-508. doi:10.1007/s00277-0162896-3

72. Kuter DJ, Mathias SD, Rummel M, et al. Health-related quality of life in nonsplenectomized immune thrombocytopenia patients receiving romiplostim or medical standard of care. Am J Hematol. 2012;87 (5):558-561

73. George JN, Mathias SD, Go RS, et al. Improved quality of life for romiplostim-treated patients with chronic immune thrombocytopenic purpura: results from two randomized, placebo-controlled trials. $\mathrm{Br}$ $J \quad H a e m a t o l$ 2009;144(3):409-415. doi:10.1111/j.13652141.2008.07464.x

74. Mathias SD, Bussel JB, George JN, McMillan R, Okano GJ, Nichol JL. A disease-specific measure of health-related quality of life for use in adults with immune thrombocytopenic purpura: its development and validation. Health Qual Life Outcomes. 2007;5 (1):11. doi:10.1186/1477-7525-5-11 


\section{Publish your work in this journal}

Patient Preference and Adherence is an international, peer-reviewed, open access journal that focuses on the growing importance of patient preference and adherence throughout the therapeutic continuum. Patient satisfaction, acceptability, quality of life, compliance, persistence and their role in developing new therapeutic modalities and compounds to optimize clinical outcomes for existing disease states are major areas of interest for the journal. This journal has been accepted for indexing on PubMed Central. The manuscript management system is completely online and includes a very quick and fair peer-review system, which is all easy to use. Visit http:// www.dovepress.com/testimonials.php to read real quotes from published authors. 